\title{
The developing paradigm of urologic training in the USA
}

\author{
Jay B. Hollander ${ }^{1}$ Chirag N. Dave ${ }^{1}$
}

Received: 9 September 2015 / Accepted: 27 October 2015 / Published online: 13 November 2015

(C) Springer Science+Business Media Dordrecht 2015

Similar to the practice of urology, modern training of urologists has undergone dramatic evolution in the last three decades. The rapid pace of change is such that without continuous learning, the well-trained surgeon of 1990 might be incompetent today. To keep pace with these changes, training programs have embraced systematic learning modules and new technology along with more conventional teaching in the operating room to successfully prepare the next generation of urologists. The best training paradigm does not exist but is in continual evolution. The US paradigm for training and maintenance of skills as it currently exists is discussed so that training programs in other countries may compare and contrast for their best practice and development.

Resident training in the USA is regulated and monitored by the Accreditation Council for Graduate Medical Education (ACGME) with each specialty having a Residency Review Committee (RRC), which establishes the requirements for certification of a residency training program. The ACGME is composed of member organizations representing physicians, hospitals and medical colleges for a coordinated program of graduated medical education. The $\mathrm{RRC}$ for urology is responsible for the monitoring and continued accreditation of urologic programs. The responsibility of running a urologic training program and the documentation of training and evaluating residents falls under a named Program Director who must be approved by the ACGME and have an appropriate academic portfolio and level of experience. The urology training program must

Chirag N. Dave

chirag.dave@beaumont.edu

1 Department of Urology, Beaumont Health System, Royal Oak, MI, USA have qualified instructors and an appropriate clinical learning environment. The spectrum of urological patients and procedures must be broad and include the multiple subspecialties of urology. The goal is to train residents to be competent to practice urology independently and without supervision within a 5- or 6-year training program. The training program includes an initial year of internship in general surgery and all residents should have completed an M.D. degree or equivalent at an accredited institution. We are in a new era of training that emphasizes professionalism, communication skills and compassion for patients. Residents, attending physicians and related medical coworkers should work in an environment of mutual respect. Such an environment was not necessarily emphasized in the past. Guidelines founded through evidence-based study and a curriculum driven by the AUA University supports a more uniform outline for resident training.

Competency-based training is now mandated and involves teaching and monitoring programs in the training of urology in six specified domains. (1) Medical Knowledge (2) Patient Care (3) System-Based Practice (4) Practice-Based Learning and Improvement (5) Interpersonal and Communication Skills and (6) Professionalism. A detailed discussion of these specific competencies is available at ACGME.org. Each resident must be evaluated with progress monitored by a competency committee formed of delegated instructors trained in evaluation. Resident progress and achievement of a competency in specific milestones of their training is documented with hope that the resident progresses toward complete competence by the end of their training. Progress in building a fund of urologic knowledge is monitored by an annual inservice exam, which simulates the experience of an actual board exam for graduates. The American public and government, which support residency training, mandate such 
competence. More details on the requirements of resident training are available at the ACGME website under the title Next Accreditation System (NAS) which is labeled as such because it is an accreditation system in continuous evolution. All urologic programs accredited by the ACGME are structured similarly with respect to mandated education. Programs must show that a didactic teaching of fundamentals of various urologic sub-specialties. Conferences must include regular morbidity and mortality conference, urologic imaging and case discussion conference and should have a urologic tumor board discussion conference. Resident duty hours are monitored with a balanced call schedule to allow residents appropriate rest and time for selflearning. Resident case logs are monitored by the RRC and proof of performance of a minimum number of indexed cases is required. All programs have mandated research requirements, which involves fundamentals of performing, presenting and publishing research projects.

State licensure to practice urology mandates proof of continuing medical education (CME) and the American Board of Urology now requires maintenance of certification (MOC) by monitoring practice patterns and fund of knowledge along with familiarity with most recent practice guidelines from organizations such as the National Comprehensive Cancer Network (NCCN), the European Association of Urology (EAU), as well as the AUA. The importance of practicing urology based on evidence-based guidelines cannot be understated. Such guidelines are generated through labor-intensive review of the literature, grading the quality of studies and meta-analysis to determine best evidence. Recommendations are made with a detailed discussion with how the recommendations were reached. The guidelines are continuously reviewed and are periodically updated. The American Urological Association (AUA) has put great efforts into making available these evidence-based guidelines, as well as best practice statements and "white paper discussions" regarding many major topics in urology. The organization has also made available to members an organized avenue for review of urology through the AUA University, which can now serve as a "textbook of urology" for urologists and residents. Furthermore, the use of hand held devices to access internet and medical websites allow modern urologists the ability to have the most sophisticated literature search on a particular medical topic at their fingertips and is encouraged among residents. Sites such as Up to Date, Pubmed, WebMD and Epocrates allow for improved patient care and daily learning by both residents and attendings.

Technology has also allowed a more robust multi modality surgical teaching environment in addition to more conventional methods of teaching in the operating room. However, the teaching faculty must continuously evolve with the technology in order to provide state of the art training.
The availability of an extensive video library online for viewing of open, endoscopic, laparoscopic, and roboticassisted procedures has clearly improved teaching and observation of technique. Simulation devices and learning centers are being developed to allow for practicing of surgical skills from simple wound closure and knot tying to advanced laparoscopic and robotic skills. Pelvic anatomy and complex reconstructive maneuvers can be learned and practiced in cadaver laboratories.

In our institution, we have developed a resident teaching paradigm to adapt to the mainstay use of robotic-assisted technologies. Residents must first familiarize themselves with laparoscopic instruments and da Vinci robot maneuvering skills on provided simulation devices in the laboratory. They then progress to observing cases and serving as bedside assistants progressing to performing portions of robotic procedures once their skills have been proven. They progress in a graded fashion performing steps of procedures until they have proven they can safely and efficiently complete an entire procedure by the end of their training. The use of a dual console in robotic procedures has been invaluable in providing safe training and observation of resident skills. Laparoscopic and robotic-assisted techniques have become the mainstay for abdominal and retroperitoneal procedures, including prostatectomy, cystectomy, nephrectomy and partial nephrectomy. The technique is so widely accepted that there may be a time where open urologic training is lacking and training programs must create new paradigms to ensure proper training of these critical skills. At our institution junior residents are trained on fundamental open surgical techniques in an animal laboratory with swine under anesthesia where maneuvers such as nephrectomy, management of the vascular pedicel, repair of vascular and ureteral injury can be simulated. This allows for the development of fundamental surgical technique allowing for safer training in the real operating room. Senior residents, in addition to their caseload, will supervise junior residents performing basic open procedures to encourage teaching and maximize exposure to open surgery.

At the conclusion of training, residents should have been exposed to a minimum of indexed cases, which covers the spectrum of general urologic practice. Residents should have achieved competence in described milestones in the basic urologic surgical domains. They should be able to practice independently and without supervision. Most important, they must practice ethically and with compassion for their patients. They must have the judgment to ask for help or assistance in difficult cases. They must know their surgical limitations and when to refer the patient for best care. Finally, they must continue to learn and develop throughout their career. Those who train urology residents understand the importance of developing judgment that 
results in patient care that is responsive to the patient's best interest even if it supersedes self-interest. The best training in my opinion is done in an environment that emphasizes resident learning over workload. The best patient care often comes from caring for the patient, and similarly, the best resident training often comes from caring for the trainees. 